\title{
A fast algorithm for manipulation control process of distribution system planning solution
}

\author{
Lin CHENG $^{1}$, Yuxiang WAN $^{1}{ }^{\mathbb{D}}$, Liting TIAN $^{1}$, Fang ZHANG $^{2}$
}

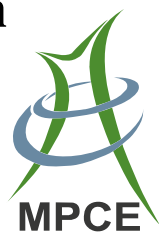

\begin{abstract}
Better flexibility and controllability have been introduced into distribution system with the development of new loads and resources. As a consequence, the connotations and tools for evaluating the planning solution need to be further enriched. This paper proposes a fast algorithm to quantify steady-state voltages and load profiles in distribution system by simulating the manipulation control process of controllable resources, taking the efficiency and ease of use into account. In this method, a complex distribution system is decoupled into several simple parts according to the ports of the DC interlink. Then, to achieve the qualified voltages and load profiles, the manipulation details of controllable resources are simulated following a certain control sequence in each part. Finally, the analysis results of each part are matched and filtered to obtain a complete evaluation. Five of the most commonly controllable resources are considered in this
\end{abstract}

CrossCheck date: 19 September 2019

Received: 29 April 2019/Accepted: 19 September 2019/Published online: 15 November 2019

(C) The Author(s) 2019

$凶$ Yuxiang WAN

wyx17@mails.tsinghua.edu.cn

Lin CHENG

chenglin@mail.tsinghua.edu.cn

Liting TIAN

tianliting@mail.tsinghua.edu.cn

Fang ZHANG

thu.zhangfang@gmail.com

1 State Key Laboratory of Control and Simulation of Power System and Generation Equipment, Department of Electrical Engineering, Tsinghua University, Beijing, China

2 School of Electrical Engineering, Beijing Jiaotong University, Beijing, China method. The effectiveness of the proposed method is demonstrated through a case study based on field data from an actual distribution system.

Keywords Controllable resources, Distribution system, DC interlink, Planning, Manipulation control process

\section{Introduction}

The increase in distributed generation (DG), electric vehicles (EVs), flexible demand, and advanced control technologies makes the distribution systems undergo profound changes regarding composition, flexibility and controllability. Meanwhile, these resources have advanced capabilities that can potentially regulate distribution systems. These advances challenge the conventional planning methodologies and bring the opportunity to enrich the evaluation ideas of planning solutions [1,2].

There are rich controllable resources in the future distribution systems, such as the distributed photovoltaic (PV), on load tap changing (OLTC) transformer, shunt capacitor and so on. Particularly, new technologies such as DC interlinks between distinct distribution feeders have been introduced to aid the management of distribution systems [3, 4]. Therefore, checking the feasibility of a candidate planning solution should rely on analyzing the manipulation control details rather than hardware redundancy alone [5]. It is a crucial step and is of fundamental interest.

However, the characteristics of modern distribution systems can be very complex due to the integration of a plethora of devices which are intermittent, distributed and interactive. Goals such as reliable power supply versus intermittent renewable sources, violated voltages caused by 
mismatching DG integration and load, and demand flexibility versus quality of service can be in conflict. Distribution system operators (DSOs) typically need to obtain the manipulation control process of these controllable resources to determine if a possible middle ground exists during the planning phase. The existing research typically focuses only on a single objective considering the collaborative planning with a few components. Reference [6] uses the site and size selection of DGs to enhance the system stability by minimizing the line losses and voltage violation. A reasonable configuration of DGs is also confirmed to delay or eliminate the expansion need triggered by load growth [7]. The DG capabilities of voltage regulation are analyzed in $[8,9]$. The coordinated control of OLTC transformer and DGs is also widely studied to control the voltage problem $[10,11]$. Further, the effect of utilizing demand response is considered to mitigate system intermittency caused by renewable energy resources in [12]. Reference [13] analyzes the impact of EVs and PVs on a low-voltage distribution network and its future planning. Reference [14] proposes a comprehensive configuration of DGs and EV charging stations to suppress renewable fluctuations based on the existing literature. In addition, the system loadability has been analyzed in the presence of DC interlinks [3, 15]. But in the future, the increasing integration of many distributed energy resources should be fully considered in the planning solution. Different from the investigation of planning work involving individual devices, an integrated evaluation method that fully considers various devices can aid in the planning work, which cannot be found in the existing literature.

On the other hand, since distribution system planning work is usually carried out by repeatedly modifying and involving the gradual enrichment of devices, the evaluation method should balance the efficiency and the optimal solution with detailed manipulation. In fact, planning problems that take the control of multiple devices into account are often difficult to solve [5]. In terms of optimization algorithm, the related research can be divided into two categories. One is heuristic calculations that favor independent models, such as particle swarm optimization [16], genetic algorithm [17], tabu search algorithm [18] and greedy algorithm [19]. The advantages of these algorithms are that they are simple and easy to operate, and can effectively obtain the approximate optimal solution, but the results are highly contingent, and the monotonicity of the results on the parameters is unstable [20]. Some other scholars are committed to framing them as an optimization model, improving the model mathematical properties, and transforming the objective function and constraint conditions into convex functions and constraints of the first and second orders. Some well-known models for the optimal power flow problems include mixed integer nonlinear programming (MINLP) [21], second-order cone programming (SOCP) [22, 23] and semi-definite programming (SDP) [24, 25]. Among them, SDP relaxation is a promising convexity method. Reference [26] utilizes this method to solve an optimal operation model of voltage regulating devices including DG and shunt capacitors. A major challenge, however, is that the optimization problems are generally large-scale, mixed-integer, non-convex even if the integral constraints are fixed, and include a large number of snapshots in time and scenarios. The low computational efficiency will bring great inconvenience to the planning work.

Motivated by this background, an algorithm is proposed for the manipulation control process of distribution system planning solution. This method decouples a complex distribution system into simple parts for evaluation according to the ports of the DC interlink. The manipulation details of controllable resources are simulated following a specific control sequence in each part to improve the efficiency. Five types of controllable resources are considered, including load tap changing (LTC) transformer, shunt capacitor, distributed PV generation, EVs, and DC interlink. Two main contributions of this paper are as follows:

1) The manipulation control of five types of devices is considered in this method. The solution results allow planners to obtain the detailed manipulations of feeders with multiple types of equipment and determine if a system configuration has acceptable voltage, load, or EV demand response profiles.

2) The proposed framework is based on testing the feasibility of candidate solutions. In particular, a sequence of simple algebraic calculations is developed to avoid solving a complex, large-scale mixed-integer optimization problem. So the computational efficiency is increased significantly. It is more applicable to the scenarios with integration of various controlled resources to distribution network, the planning of which has the features of screening a large number of planning schemes.

The proposed algorithm is demonstrated by considering an actual distribution system that consists of two sub-systems tied with a DC interlink, each of which contains high penetration of distributed PVs and EVs. The detailed manipulation and impact of these devices can be captured by the proposed method, and the benefit of the DC interlink is shown. In addition, the computation complexity of the proposed algorithm and optimization based planning methods is compared. The results show that the proposed algorithm reduces the computational time significantly.

The rest of the paper is organized as follows. The planning problem is discussed and the structure of the proposed algorithm, as well as some utilized tools, is 
briefly explained in Section 2 . Then the specific procedure is introduced in Section 3. The proposed algorithm is tested using a case system based on an actual distribution system in Section 4. Finally, this paper is concluded in Section 5.

\section{Planning problem and solution methodology}

This section shows the common configuration of future distribution systems and sets up the planning problem. The structure of the solution methodology is briefly explained accordingly. And some mathematical preliminaries in the simulation are introduced.

\subsection{Planning problem formulation}

Future distribution systems experience rapid growth of load, distributed PV and EV, and may install some DC interlinks for load sharing or voltage support. A possible configuration of such a system is shown in Fig. 1.

In these systems, because of the growing high-density load and the transferred load with the $\mathrm{N}-1$ contingency, the voltage of urban district might be too low, and some transformers suffer overload risk. But at the end of some feeders with high PV penetration, the voltage of the suburban district might be abnormally high. The conventional planning approach to correct these problems is to expand and upgrade the substation, which may be prohibitively costly. But the costs can be minimized by strategically utilizing the controllable devices like LTC transformers, capacitors, PV inverters, EVs and DC interlinks.

In distribution system planning work, the goal is to determine whether these controllable devices can be used to satisfy various operation constraints or whether new equipment is required. The planners might make several candidate solutions, then run a series of evaluations to estimate whether these problems such as overload,

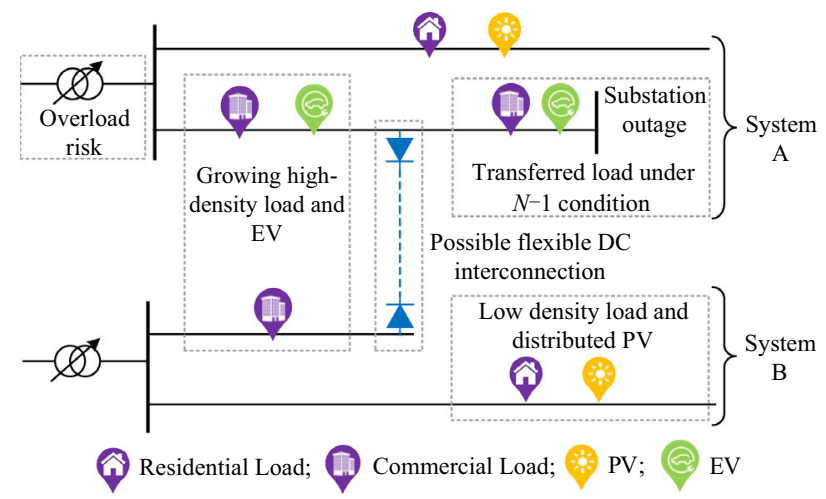

Fig. 1 A possible configuration of the future distribution system including LTC transformers, capacitors, EVs, DGs, PVs and a DC interlink overvoltages, renewable spillage, and load curtailment can be solved.

\subsection{Solution methodology}

The proposed algorithm focuses on checking the feasibility from the aspects of load and voltage profiles. For this purpose, the method evaluates if a candidate solution would have acceptable voltage, load, and load curtailment profiles by simulating the manipulation control process.

In order to carry out the evaluation efficiently, the overall problem is divided into a series of small ones and solved in sequence. The procedure is outlined in Fig. 2. In a complex system with a DC interlink, we first decouple it into separate parts, check the feasibility of the solution for each part, and then use the DC interlinks to balance the smaller systems.

The manipulation simulation follows the general guiding principle in which discretely adjustable equipment is prioritized over continuously regulating equipment. The reactive equipment is prioritized over active equipment, and power regulation is prioritized over load regulation [27]. Therefore, this method allows planners to determine if a system configuration has acceptable voltage, load, or

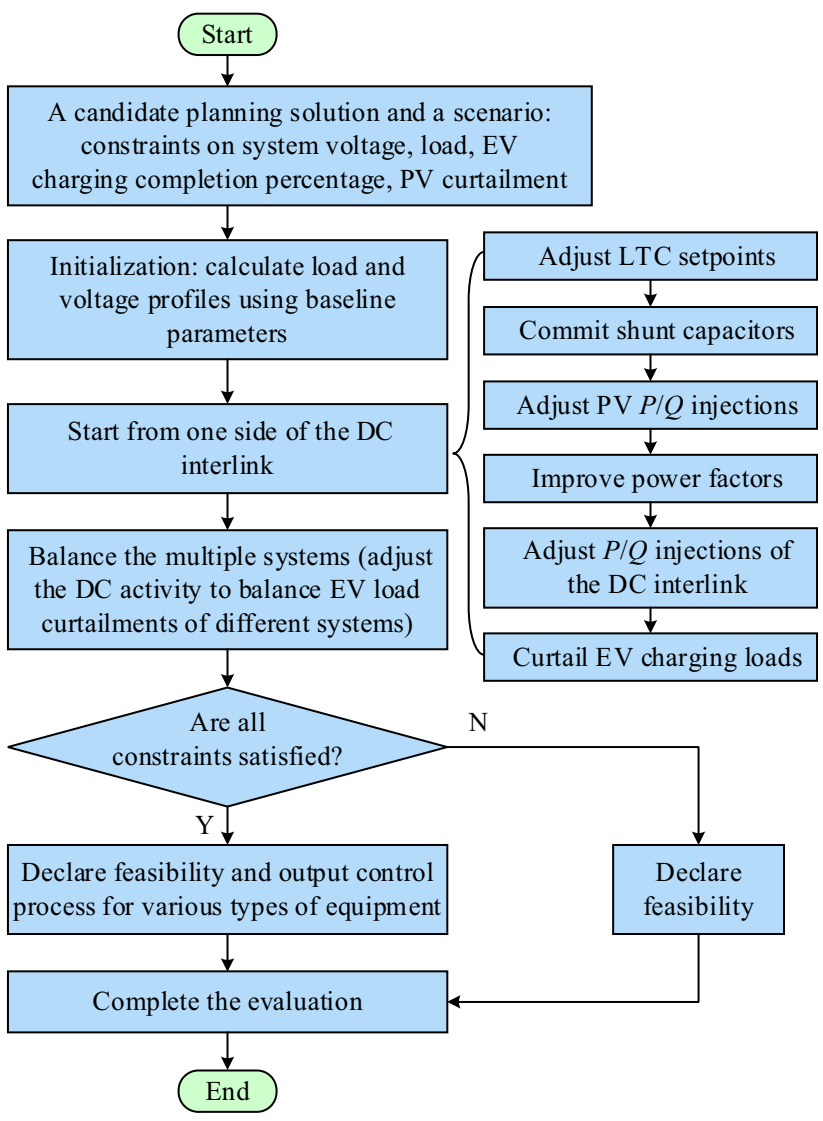

Fig. 2 Main algorithm to evaluate a candidate solution 
EV demand response profiles in a computationally tractable fashion and provides detailed manipulation simulation of feeders with multiple types of equipment.

\subsection{Power flow calculation tools}

In the evaluation procedure of Fig. 2, some mathematical preliminaries are applied such as the flow sensitivity, the optimal partitioning method, the second-order power loss sensitivity, and the EV charging strategy. Among them, power flow calculation and flow sensitivity are widely used. This subsection is a brief introduction to the second-order power loss sensitivity, the optimal partitioning method, and the EV charging strategy.

\subsubsection{Second-order power loss sensitivity}

The loss should be considered when the capacity problem is studied. An important metric we use to determine the adjustment of the devices is based on the losses in the system. Because of the nonlinear nature of power flow in distribution system, we adopt the second-order power loss sensitivity method proposed in [28] for both active power and reactive power:

$$
\begin{aligned}
\boldsymbol{G}_{P L}(\Delta \boldsymbol{P}, \Delta \boldsymbol{Q})= & \boldsymbol{P}_{\text {Loss }, 0}+\boldsymbol{\alpha}_{P P}^{\mathrm{T}} \Delta \boldsymbol{P}+\boldsymbol{\alpha}_{P Q}^{\mathrm{T}} \Delta \boldsymbol{Q} \\
& +\frac{1}{2}\left[\begin{array}{ll}
\Delta \boldsymbol{P}^{\mathrm{T}} & \Delta \boldsymbol{Q}^{\mathrm{T}}
\end{array}\right] \boldsymbol{\beta}_{P}\left[\begin{array}{c}
\Delta \boldsymbol{P} \\
\Delta \boldsymbol{Q}
\end{array}\right] \\
\boldsymbol{G}_{Q L}(\Delta \boldsymbol{P}, \Delta \boldsymbol{Q})= & \boldsymbol{Q}_{\text {Loss }, 0}+\boldsymbol{\alpha}_{Q P}^{\mathrm{T}} \Delta \boldsymbol{P}+\boldsymbol{\alpha}_{Q Q}^{\mathrm{T}} \Delta \boldsymbol{Q} \\
& +\frac{1}{2}\left[\begin{array}{ll}
\Delta \boldsymbol{P}^{\mathrm{T}} & \Delta \boldsymbol{Q}^{\mathrm{T}}
\end{array}\right] \boldsymbol{\beta}_{Q}\left[\begin{array}{c}
\Delta \boldsymbol{P} \\
\Delta \boldsymbol{Q}
\end{array}\right]
\end{aligned}
$$

where $\boldsymbol{G}_{P L}(\cdot)$ and $\boldsymbol{G}_{Q L}(\cdot)$ are the incremental active power and reactive power $(P / Q)$ loss functions of nodal $P / Q$ injection increments, respectively; $\boldsymbol{\alpha}_{P P}, \boldsymbol{\alpha}_{P Q}, \boldsymbol{\alpha}_{Q P}, \boldsymbol{\alpha}_{Q Q}$, $\boldsymbol{\beta}_{P}, \boldsymbol{\beta}_{Q}$ are the coefficients calculated from the power flow equations which can be seen in [28]; $\Delta \boldsymbol{P}$ and $\Delta \boldsymbol{Q}$ are the node active and reactive power injection increments, respectively; and $\boldsymbol{P}_{\text {Loss }, 0}$ and $\boldsymbol{Q}_{\text {Loss }, 0}$ are the active and reactive loss scalars of the system in the reference state, respectively.

\subsubsection{Optimal partitioning method for LTC transformers and capacitors}

During the evaluation of a solution, the first equipment to be adjusted should be discrete elements such as LTC transformers and capacitors which are subject to daily manipulation limits stemming from lifespan considerations. Our proposed algorithm solves this question via the optimal partitioning technique. The algorithm can divide a series of data into a designated number of segments based on maximum homogeneity. The similarity of system voltage profiles within a segment of time suggests little need to change the settings of LTC transformer and capacitor. That is, for all time snapshot $t$ in a time segment $s$, their settings should be fixed. More details about the optimal partitioning technique can be found in [29].

\subsubsection{EV charging strategy}

The charging of EVs can be adjusted to relieve system stress. The reliability index of charging service proposed in [30] is used to assess how EVs would be affected as flexible loads. It would be a key performance indicator when distribution system planning is concerned. Charging completion is defined as:

$\varepsilon=\left\{\begin{array}{cc}1 & \sum_{t \in\left[t_{\text {arr }}, t_{\text {dep }}\right]} p_{\mathrm{c}, t} \Delta t-\alpha E_{\mathrm{D}} \geq 0 \\ 0 & \text { otherwise }\end{array}\right.$

It means that the total energy charged to a vehicle should exceed a certain percentage $\alpha$ of the expectation of user $E_{\mathrm{D}}$ during the arrival time $t_{\text {arr }}$ to the departure time $t_{d e p}$. In order to solve the indicator $\varepsilon$, it is necessary to calculate the charging process $p_{\mathrm{c}, t}$. Let $p_{\mathrm{c}, k, t}$ denote the charging power of vehicle $k$ at time $t$, and $\bar{p}_{\text {rate }}$ is the maximum charging power. The system imposes a limit on the overall charging power of the EVs, denoted by $P_{\mathrm{EV}, t}$, and the charging profiles are optimized against this external limit.

$\max \sum_{t \in[0, T]} \sum_{k \in \Omega} p_{\mathrm{c}, k, t} \Delta t$

s.t.

$\sum_{k \in \Omega} p_{\mathrm{c}, k, t} \Delta t \leq P_{\mathrm{EV}, t} \quad \forall t \in[0, T]$

$\sum_{t \in\left[t_{\text {arr }, k}, t_{\text {dep }, k}\right]} p_{\mathrm{c}, k, t} \Delta t \leq E_{\mathrm{D}, k} \quad \forall k \in \Omega$

$0 \leq p_{\mathrm{c}, k, t} \leq \bar{p}_{\text {rate }} \quad \forall k \in \Omega, \forall t \in[0, T]$

where $T$ is a time segment; and $\Omega$ is the set of $\mathrm{EV}$ stations.

Formula (4) maximizes the total energy consumed by the EVs, (5) is the total power constraint, (6) is the constraint that EVs are not charged more than their energy demand $E_{\mathrm{D}, k}$, and (7) is the individual EV charging power constraints. All the parameters except $\Delta P_{\mathrm{EV}, t}$ are predefined in the data scenarios, while the parameter $P_{\mathrm{EV}, t}$ is solved by subtracting power curtailment $\Delta P_{\mathrm{EV}, t}$ from the maximum charging power $\underline{P}_{\mathrm{EV}, t}$ after balancing $\mathrm{EV}$ load curtailments in Fig. 2. 


\section{Simulation of manipulation control process}

This section introduces the specific implementation procedure in Fig. 2. In the following section, the detailed manipulation control process with five types of equipment can be obtained considering the operation constraints.

\subsection{Initialization of baseline system status}

Given a scenario with sequential load, PV generation $\bar{P}_{\mathrm{PV}, t}$ and EV charging profiles $\underline{P}_{\mathrm{EV}, t}$, the first step is to calculate the baseline system status. By baseline, we mean the power flow with the given load and PV generation as injections, while LTC transformer tap is set to 1.0 p.u., and there are no additional injections from capacitors and DC terminals.

According to the baseline nodal voltage profiles, the given scenario day is then partitioned into several time segments for possible LTC transformer and capacitor adjustments via the optimal partitioning technique. The voltage and loss sensitivities are also calculated according to this baseline.

\subsection{Simulation of one subsystem connected by DC interlink}

Our proposed algorithm starts from one subsystem that is connected to another subsystem by a DC interlink, as shown in Fig. 1. Each equipment is adjusted in turn, as shown in Fig. 2. The voltage and load profiles need to be updated after each step.

\subsubsection{Simulation of LTC transformer tap setting}

The LTC transformer has a decisive effect on voltage regulation and its setting is determined first. A tap position (modeled as the incremental of secondary voltage setpoint $\left.\Delta \rho_{A, T}\right)$ should be able to keep the voltages on all feeders being within the voltage limits in a time segment, expressed as:

$V_{\min }-V_{i, t} \leq \Delta \rho_{A, T} \leq V_{\max }-V_{i, t} \quad i \in f, \forall f \in A, \forall t \in T$

where $V_{\min }$ and $V_{\max }$ are the limits of voltage magnitude; $i \in f$ denotes node $i$ belonging to feeder $f$; and $\forall f \in A$ denotes feeder $f$ belonging to system A.

There may not be tap settings that satisfy (8) for all feeders at all times. Considering that the controllable sources can be used to assist voltage regulation, we can still determine a tap setting in advance, and compensate the voltage gaps in the remaining steps in Fig. 2. That is, the tap ranges can be virtually extended and the values are given by (8) using power flow sensitivities.

$$
\begin{gathered}
V_{\min }-\left[V_{i, t}+\sum_{i, j \in f}\left(K_{i j} \Delta \underline{Q}_{j, t}+H_{i j} \Delta \underline{P}_{j, t}\right)\right] \leq \Delta \rho_{A, T} \\
\leq V_{\max }-\left[V_{i, t}+\sum_{i, j \in f}\left(K_{i j} \Delta \bar{Q}_{j, t}+H_{i j} \Delta \bar{P}_{j, t}\right)\right]
\end{gathered}
$$

where $\Delta \underline{P}_{j, t}, \Delta \bar{P}_{j, t}, \Delta \underline{Q}_{j, t}, \Delta \bar{Q}_{j, t}$ are the lower and upper bounds of additional injections, respectively, which are generated assuming committing all capacitor groups, curtailing all charging loads etc.; $H_{i j}$ and $K_{i j}$ are the power flow sensitivities, respectively; and $V_{i, t}$ is the voltage of node $i$ at time $t$.

The left side of (9) means that power injection or load curtailment provide additional adequacy to lower LTC transformer secondary setpoint, and vice versa. For the DC terminal, the best $P / Q$ injection ratio for regulating voltage is $H_{i j} / K_{i j}$. For PVs, the bound of the adjustable active power injection is $\Delta \underline{P}_{j, t}=-\bar{P}_{\mathrm{PV}, j, t}$ and $\Delta \bar{P}_{j, t}=0$, where $\bar{P}_{\mathrm{PV}, j, t}$ is the PV active power generation of node $j$ at time $t$.

By repeating (9) for all snapshots in time, a series of tap ranges can be generated, and the tap setting should be chosen from their intersection. When there are multiple tap setting choices, we prefer the candidate with the least requirement on active power $\underline{P}_{j, t}$ and $\bar{P}_{j, t}$. With the LTC transformer tap set to an acceptable value, the following procedures calculate precisely how much power is required from each controllable source for voltage regulation.

\subsubsection{Simulation of shunt capacitor}

In this step, the shunt capacitors are committed to compensating the under-voltage left over by LTC transformer. For a feeder $f$, let $\Delta C_{\mathrm{CP}, j}=C_{\mathrm{CP}, j} / N_{\mathrm{CP}, j}$ represent the reactive power of each capacitor group, where $C_{\mathrm{CP}, j}$ is the capacity of shunt capacitor $j$ and $N_{\mathrm{CP}, j}$ is the group number of shunt capacitor $j$, and let $\Delta V_{i, t}=$ $\max \left\{V_{\min }-V_{i, t}, 0\right\}$ represent the level of under-voltage. We need to solve a reactive power injection $\Delta Q_{\mathrm{CP}, j, T}$ which satisfies (10). The equation considers voltage correction $\Delta V_{i, t}$ and capacity $C_{\mathrm{CP}, j}$. The discreteness of capacitors is enforced by the ceiling operator.

$$
\begin{array}{r}
\Delta Q_{\mathrm{CP}, j, T}=\Delta C_{\mathrm{CP}, j}\left[\frac{1}{\Delta C_{\mathrm{CP}, j}} \min \left\{\frac{\Delta V_{i, t}}{K_{i j}}, C_{\mathrm{CP}, j}\right\}\right] \\
i \in f, j \in f \cap S_{\mathrm{CP}}, \forall t \in T
\end{array}
$$

where $S_{\mathrm{CP}}$ is the set of nodes with shunt capacitors.

When there are multiple capacitors on a feeder, each capacitor is calculated according to the descending order of $K_{i j}$. 


\subsubsection{Simulation of reactive power of $P V$ inverter}

In this step, the reactive power from PV inverters are committed to compensate either the under-voltage left over by the LTC transformers and capacitors, or the overvoltage left over by LTC transformer. The inverter has a reactive power capability to operate within a power factor range, e.g. [ $-0.95,0.95]$. The potential of PV inverter for adjusting voltage is significant in the system. For a feeder $f$, the under-voltage problem is solved via:

$$
\begin{array}{r}
\Delta Q_{\mathrm{PV}, j, t}=\min \left\{\frac{V_{\min }-V_{i, t}}{K_{i j}}, \frac{\sqrt{1-0.95^{2}} C_{\mathrm{PV}, j, t}}{0.95}\right\} \\
i \in f, j \in f \cap S_{\mathrm{PV}}
\end{array}
$$

where $S_{\mathrm{PV}}$ is the set of nodes with distributed PVs; $\Delta Q_{\mathrm{PV}, j, t}$ is the PV reactive power of node $j$ at time $t$; and $C_{\mathrm{PV}, j, t}$ is the rated capacity of PV generator $j$ at time $t$.

The items in the bracket of (11) are the reactive power required for voltage correction, and the reactive power capacities under power factor constraints. For overvoltage, it is corrected via:

$$
\Delta Q_{\mathrm{PV}, j, t}=\max \left\{\frac{V_{\mathrm{max}}-V_{i, t}}{K_{i j}},-\frac{\sqrt{1-0.95^{2}} C_{\mathrm{PV}, j, t}}{0.95}\right\}
$$

When there are multiple PVs on a feeder, each PV is calculated sequentially according to the descending order of $K_{i j}$.

\subsubsection{Simulation of PV curtailment}

Meanwhile, a portion of PV power has to be curtailed when an overvoltage on a feeder $f$ still exists. Then PV power curtailments $\Delta P_{\mathrm{PV}, j, t}$ is calculated via:

$$
\Delta P_{\mathrm{PV}, j, t}=\max \left\{\frac{V_{\mathrm{max}}-V_{i, t}}{H_{i j}},-\bar{P}_{\mathrm{PV}, j, t}\right\} \quad i \in f, j \in f \cap S_{\mathrm{PV}}
$$

\subsubsection{Improvement of power factor}

The capacitors and PV inverters can improve LTC transformer power factor and relieve load if voltage problem no longer exists on their feeders. For example, capacitors should be re-adjusted if the system has a significant overall reactive load surplus. The adjustment is calculated as:

$$
\begin{aligned}
& \Delta Q_{\mathrm{CP}, j, T} \\
& =\Delta C_{\mathrm{CP}, j}\left\lceil\frac{1}{\Delta C_{\mathrm{CP}, j}} \min \left\{\frac{V_{\mathrm{max}}-V_{i, t}}{K_{i j}}, C_{\mathrm{CP}, j}-Q_{\mathrm{CP}, j, T}, Q_{\mathrm{tot}, t}\right\}\right] \\
& t \in T, i \in f, \forall f \in A, \forall j \in f \cap S_{\mathrm{CP}}
\end{aligned}
$$

The items in the bracket are the voltage adequacy for accommodating more reactive power, the remaining capacity of the capacitor, and the overall system reactive load surplus $Q_{\text {tot }, t}$, respectively. If there are multiple sources on a feeder, the source is calculated sequentially according to the ascending order of $K_{i j}$. The PVs share similar calculations.

\subsubsection{Simulation of $P / Q$ injections of $D C$ interlink}

If voltage and load problems still exist after the procedures mentioned above, we use active and reactive power injections from the DC terminal. We need to solve a combination of $\Delta P_{\mathrm{DC}, t}, \Delta Q_{\mathrm{DC}, t}$ with respect to converter capacity, voltage and LTC transformer load corrections via:

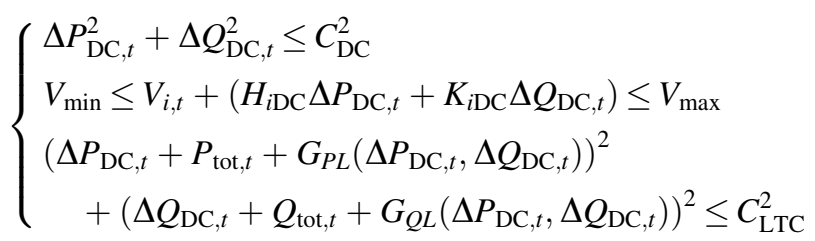

where $P_{\mathrm{DC}, t}$ and $Q_{\mathrm{DC}, t}$ are the $P / Q$ injections of DC terminal; $C_{\mathrm{DC}}$ is the capacity of a DC terminal; $P_{\text {tot }, t}$ is the overall active power of an OLTC transformer; $C_{\mathrm{LTC}}$ is the capacity of an OLTC transformer; $H_{i \mathrm{DC}}$ and $K_{i \mathrm{DC}}$ are the power flow sensitivities of DC terminal; and $G_{P L}(\cdot)$ and $G_{Q L}(\cdot)$ are the incremental $P / Q$ loss functions of nodal $P / Q$ injection increments.

The (15) represents constraints on the apparent power capacity of the DC interlink, correction of voltage problems, and the constraint that the apparent power should be less than the capacity of the LTC transformer after the effect of DC injections on power losses are added to LTC transformer load.

Though it is difficult to derive an analytical answer directly, it is only a two-dimensional problem which can be solved easily by exhaustive search, representing the first constraint of (15) done by a series of coordinates. Then the solutions are exhaustively searched to see if they can meet the other two constraints. If all the constraints can be satisfied, the coordinate with a minimum absolute value of $\Delta P_{\mathrm{DC}, t}$ will be selected since it imposes the least impact on the other side of the DC interlink. Otherwise, the 
coordinate with minimum voltage violation or overload will be selected.

\subsubsection{Simulation of EV curtailment}

Curtailing EV charging loads are needed in case of persistent under-voltage or overload. Our strategy attempts to ensure that no EV charging site suffers outstanding curtailment in order to avoid unnecessary charging incompletion. The curtailment is solved in two parts. The first part relieves the under-voltage using charging sites on the same feeder.

$$
\left\{\begin{array}{l}
\Delta P_{\mathrm{EV}, j, t}=\lambda_{\mathrm{I}, j, t} \underline{P}_{\mathrm{EV}, j} \quad \forall j \in f \cap S_{\mathrm{EV}} \\
\lambda_{\mathrm{I}, f, t}=\min \left\{\left(V_{\min }-V_{i, t}\right) / \sum H_{i j} \underline{\mathrm{EV}}, j, t_{1}, 1\right\} \quad i \in f
\end{array}\right.
$$

where $\Delta P_{\mathrm{EV}, j, t}$ is the curtailments of $\mathrm{EV}$ charging power at a charging site; $\underline{P}_{\mathrm{EV}, j}$ is the maximum charging power (minimum injection) of a charging site; and $S_{\mathrm{EV}}$ is the set of nodes with EV charging sites.

The first equation in (16) enforces that the EV charging sites on the same feeder will have the same curtailment ratio $\lambda_{\mathrm{I}, f, t}$ (between 0 and 1 ). Then $\lambda_{\mathrm{I}, f, t}$ is solved to correct the under-voltage of $V_{\min }-V_{i, t}$.

The second equation in (16) relieves the overload using a curtailment ratio $\lambda_{\mathrm{II}, j, t}$. Since some charging sites have taken responsibilities to relieve under-voltage, further duties should be avoided for these sites. So the curtailment in this stage starts from those who have intact charging loads, and $\lambda_{\mathrm{II}, j, t}$ is solved based on the following steps.

Step 1: The baseline values of $\lambda_{\mathrm{II}, j, t}$ are $\lambda_{\mathrm{I}, f, t}, j \in f$.

Step 2: Decide the feeder $f$ which has the second smallest $\lambda_{\mathrm{I}, f, t}$.

Step 3: For those charging sites whose $\lambda_{\mathrm{II}, j, t}$ is smaller than $\lambda_{\mathrm{I}, f, t}$, enumerate a scalar $\Delta \lambda_{\mathrm{II}, j, t}$ so that $\lambda_{\mathrm{II}, j, t}+\Delta \lambda_{\mathrm{II}, j, t}$ satisfies (17). The value is obtained by one-dimensional search within $\left[\lambda_{\mathrm{II}, j, t}, \lambda_{\mathrm{I}, f, t}\right]$.

$$
\left\{\begin{array}{l}
\Delta P_{\mathrm{EV}, j, t}=\lambda_{\mathrm{II}, j, t} \underline{P}_{\mathrm{EV}, j, t} \quad \forall j \in A \cap S_{\mathrm{EV}} \\
\left(P_{\mathrm{tot}}\left(\Delta P_{\mathrm{EV}, t}\right)+G_{P L}\left(\Delta P_{\mathrm{EV}, t}\right)\right)^{2} \\
+\left(Q_{\mathrm{tot}}+G_{Q L}\left(\Delta P_{\mathrm{EV}, t}\right)\right)^{2} \leq C_{\mathrm{LTC}}^{2}
\end{array}\right.
$$

The first equation in (17) suggests that the charging sites involved have the same curtailment ratio, and the second equation models the LTC transformer load constraint.

Step 4: Update $\lambda_{\mathrm{II}, j, t}$ with $\Delta \lambda_{\mathrm{II}, j, t}$. Go back to Step 2 until the overload is eliminated or each $\lambda_{\mathrm{II}, j, t}$ equals to 1 .

\subsection{Modeling of the other side of DC interlink}

The other side of the system in Fig. 1, i.e. system B, has the similar simulation procedures. But the active power balance requirement of DC interlink demands $P_{\mathrm{DC}, t}^{A}+P_{\mathrm{DC}, t}^{B}=0$. There will be real power loss on the DC interlink, which is not discussed in this paper. Therefore, the way of handling the DC terminal at in system B is different, as given in the following steps.

1) Initialization: instead of setting $P_{\mathrm{DC}, t}^{B}=0$, setting $P_{\mathrm{DC}, t}^{B}=-P_{\mathrm{DC}, t}^{A}$.

2) Simulating LTC transformer tap: as the active power injection is fixed, only reactive power, i.e. $\Delta \bar{Q}_{\mathrm{DC}, t}, \Delta \underline{Q}_{\mathrm{DC}, t}$ are considered in (9).

3) Simulating the $P / Q$ injections of DC interlink: only reactive power can be adjusted in (15).

\subsection{Balancing of multiple systems}

When multiple systems have under-voltage or overload problems, the first system may take advantage of other systems via the DC interlinks simply because it is regulated first. Here we illustrate how to balance the systems using a two-system network where unbalanced EV curtailment occurs. Balancing multiple systems proceeds similarly. Suppose in two systems A and B as shown in Fig. 3, the curtailment ratio $\max \left\{\lambda_{\mathrm{II}, t}^{A}\right\} \ll \max \left\{\lambda_{\mathrm{II}, t}^{B}\right\}$, where $\lambda_{\mathrm{II}, t}^{A}$ and $\lambda_{\mathrm{II}, t}^{B}$ are the ratios of $\mathrm{EV}$ charging curtailment for relieving overload in systems A and B. It is better to balance

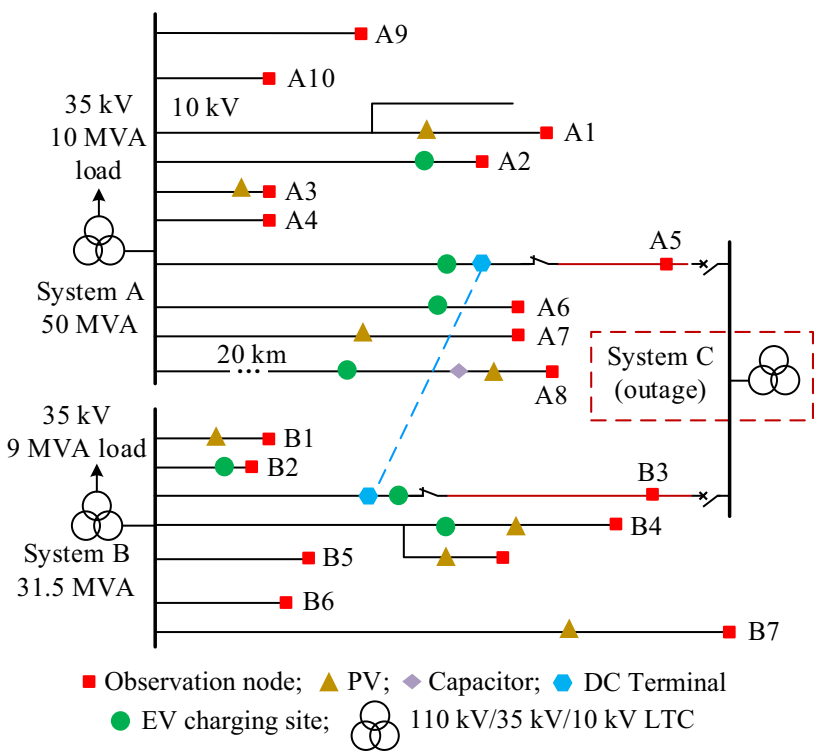

Fig. 3 Single line diagram of case system 
$\max \left\{\boldsymbol{\lambda}_{\mathrm{II}, t}^{A}\right\} \approx \max \left\{\boldsymbol{\lambda}_{\mathrm{II}, t}^{B}\right\} \quad$ in order to avoid possible uncompleted charging.

\subsubsection{Adjustment of EV curtailment and DC injection of system $B$}

The procedure starts with searching for a scalar $\Delta \lambda^{B}$ to offset the existing curtailment ratios $\lambda_{\mathrm{II}}^{B}$. For the feeder with the DC terminal in system $\mathrm{B}, \lambda_{\mathrm{II}, j, t}^{B}$ is reduced by $\Delta \lambda^{B}$. For a feeder $f$ without the DC terminal in system $B, \Delta \lambda_{\mathrm{II}, j, t}^{B}$ is calculated based on voltage adequacy.

$\Delta \lambda_{\mathrm{II}, j, t}^{B}=\min \left\{\frac{V_{i, t}-V_{\mathrm{min}}}{\sum_{j \in f \cap S_{\mathrm{EV}}^{B}} \underline{P}_{\mathrm{EV}, j, t} H_{i j}}, \Delta \lambda^{B}, \lambda_{\mathrm{II}, j, t}^{B}\right\}$

where $S_{\mathrm{EV}}^{B}$ is the set of nodes with EV charging sites in system B.

The reduced curtailments may lead to low voltage on the feeder with the DC interlink or overload on the LTC transformer. The problems will be then compensated by reducing active power consumption of the DC terminal. We can use (15) to recalculate $\Delta P_{\mathrm{DC}, t}^{B}, \Delta Q_{\mathrm{DC}, t}^{B}$ after updating the EV loads.

\subsubsection{Adjustment of DC injections and EV curtailment of system $A$}

Go back to feeder $f$ with the DC terminal in system A. Firstly, let $\Delta P_{\mathrm{DC}, t}^{A}=-\Delta P_{\mathrm{DC}, t}^{B}$, and voltage or overload problem may appear. Then the reactive power $\Delta Q_{\mathrm{DC}, t}^{A}$ of DC interlink should be updated using (19).

$$
\begin{aligned}
& \Delta Q_{\mathrm{D} C, t}^{A}=\min \left\{\Delta Q_{\mathrm{D} C, t}^{A^{\prime}}, \Delta Q_{\mathrm{D} C, t}^{A^{\prime \prime}}, \Delta Q_{\mathrm{D} C, t}^{A^{\prime \prime \prime}}\right\} \\
& \left\{\begin{array}{l}
\Delta Q_{\mathrm{DC}, t}^{A^{\prime}}=\max \left\{0,\left(V_{\min }-V_{i, t}\right) / K_{i \mathrm{DC}}\right\} \quad i \in f \\
\Delta Q_{\mathrm{DC}, t}^{A^{\prime \prime}}=-Q_{\mathrm{tot}, t}^{A} \\
\Delta Q_{\mathrm{DC}, t}^{A^{\prime \prime \prime}}=\sqrt{C_{\mathrm{DC}}^{2}-\left(P_{\mathrm{DC}, t}^{A}+\Delta P_{\mathrm{DC}, t}^{A}\right)^{2}}-Q_{\mathrm{DC}, t}^{A}
\end{array}\right.
\end{aligned}
$$

where $Q_{\text {tot }, t}^{A}$ is the overall system reactive load surplus in system A.

The first equation calculates the reactive power required for restoring voltage, the second improves the power factor, and the third models the capacity limit of the DC. After updating the DC interlink, we can refer to (16) and (17) for a new $\lambda_{\mathrm{II}, t}^{A}$.

\subsubsection{Choosing of the best adjustment}

A table of new $\max \left\{\boldsymbol{\lambda}_{\mathrm{II}, t}^{A}\right\}$ and $\max \left\{\boldsymbol{\lambda}_{\mathrm{II}, t}^{B}\right\}$ can be generated by enumerating a series of values of $\Delta \lambda^{B}$ and repeating the procedure. The step minimizing the gap between $\max \left\{\lambda_{\mathrm{II}, t}^{A}\right\}$ and $\max \left\{\lambda_{\mathrm{II}, t}^{B}\right\}$ is then chosen to update DC interlink injections and EV curtailments. Finally, the outputs, setpoints of all controllable sources as well as system voltage and load profiles can be obtained.

\section{Case studies}

\subsection{Case introduction}

The case originates from an actual distribution system in Beijing, China. The single line diagram is shown in Fig. 3. A rapid annual load growth of this system is around $10 \%$. Especially, the EV fleet grows at $100 \%$ per year. The majority of load gathers around the center, while the outer area has scattered loads powered by long feeders. The abundant land also makes the outer areas be good places for PV panels. The transformers adopt LTC transformer to regulate voltage with \pm 8 grades and each grade is adjusted by 0.0125 p.u.. Some capacities of the transformers are occupied by the $35 \mathrm{kV}$ system. The available capacities of the transformers of system A and system B are 40 MVA and 22.5 MVA, respectively.

Assuming that the configuration is for a distribution system planning solution, the sub-systems have overvoltage and overload problems when an adjacent $110 \mathrm{kV}$ transformer (i.e., system C) fails and a portion of its load is transferred. The load profiles of the LTC transformers and voltage profiles of typical feeders are shown in Fig. 4. Without consideration of the manipulation control process,
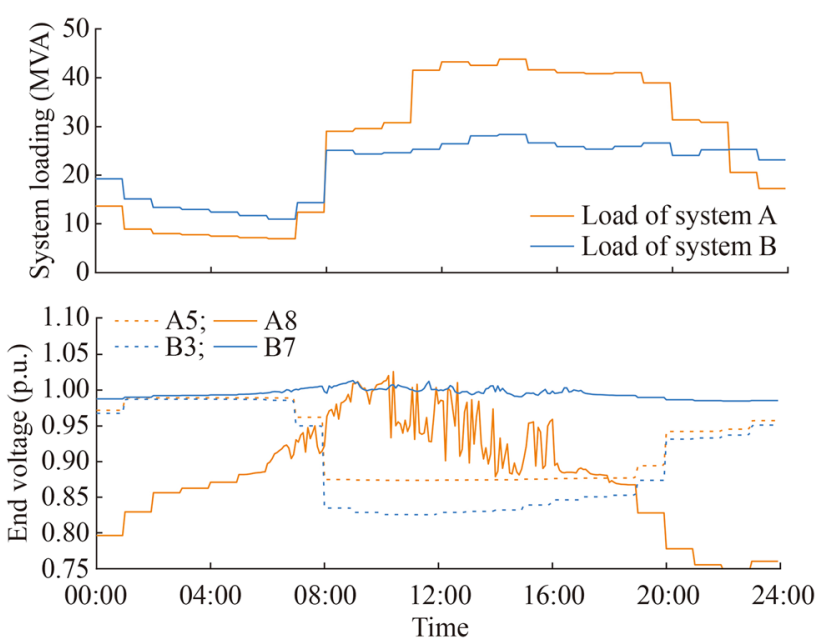

Fig. 4 Profiles of baseline load and voltage of case systems 
the evaluation results mean that additional expansions are required. However, the controllability and flexibility are supposed to be introduced into the demonstration area, like fully utilizing the adjustable potential of EVs or the reactive power support of PV inverters. Given the complicated voltage and load problems and various controllable sources, it is not trivial for planners to decide whether this solution is sufficient, or more extensive expansions of the network are needed. The proposed solving method for the manipulation control process of a planning solution can be applied to aid this decision.

\subsection{Simulation results}

First, it will demonstrate how the proposed algorithm in Fig. 2 is applied. For example, systems A and B have overload and voltage violation risks at 12:10 p.m.. And the

Table 1 Simulated events at 12:10 p.m.

\begin{tabular}{|c|c|c|}
\hline $\begin{array}{l}\text { Simulation } \\
\text { process }\end{array}$ & System A & System B \\
\hline Initial load & $40.05 \mathrm{MVA}$ & 25.0 MVA \\
\hline Initial voltage & $\begin{array}{l}\text { A5 voltage is } 0.86 \text { p.u., A8 } \\
\text { voltage is } 1.07 \text { p.u. }\end{array}$ & $\begin{array}{l}\text { B3 voltage is } 0.83 \\
\text { p.u., B7 voltage } \\
\text { is } 1.00 \text { p.u. }\end{array}$ \\
\hline $\begin{array}{l}\text { LTC } \\
\text { transformer } \\
\text { secondary } \\
\text { setpoint }\end{array}$ & +0.05 p.u. & +0.0625 p.u. \\
\hline Shunt capacitor & A8: no group & - \\
\hline $\begin{array}{l}\mathrm{PV} \text { reactive } \\
\text { power }\end{array}$ & A8: consumes 0.45 Mvar & - \\
\hline $\begin{array}{l}\text { PV active and } \\
\text { reactive } \\
\text { power }\end{array}$ & $\begin{array}{l}\text { A8: curtails } 0.28 \mathrm{MW} \text { and } \\
\text { consumes } 0.42 \mathrm{Mvar}\end{array}$ & - \\
\hline $\begin{array}{l}\text { Increase power } \\
\text { factor }\end{array}$ & $\begin{array}{l}\text { Capacitor does not change } \\
\text { and } 0.87 \text { Mvar reactive } \\
\text { power is injected from PVs }\end{array}$ & $\begin{array}{l}0.7 \text { Mvar reactive } \\
\text { power is } \\
\text { injected from } \\
\text { PVs }\end{array}$ \\
\hline $\begin{array}{l}\text { DC active and } \\
\text { reactive } \\
\text { power }\end{array}$ & $-1.4 \mathrm{MW}+1.02 \mathrm{Mvar}$ & $\begin{array}{l}\text { 1.4 MW + } 0.92 \\
\text { Mvar }\end{array}$ \\
\hline $\begin{array}{l}\mathrm{EV} \text { for voltage } \\
\text { management }\end{array}$ & - & - \\
\hline $\begin{array}{l}\mathrm{EV} \text { for load } \\
\text { management }\end{array}$ & - & $\begin{array}{l}\text { System-wide: } 30 \% \\
\quad \text { curtailed }\end{array}$ \\
\hline $\begin{array}{l}\text { Readjust EV } \\
\text { curtailment }\end{array}$ & System-wide: $10 \%$ curtailed & $\begin{array}{l}\text { System-wide: } 15 \% \\
\quad \text { curtailed }\end{array}$ \\
\hline $\begin{array}{l}\text { Readjust DC } \\
\text { injection }\end{array}$ & $-2.8 \mathrm{MW}+0.9 \mathrm{Mvar}$ & $\begin{array}{l}2.8 \mathrm{MW}+0.9 \\
\text { Mvar }\end{array}$ \\
\hline Final load & $39.2 \mathrm{MVA}$ & $22.5 \mathrm{MVA}$ \\
\hline Final voltage & $\begin{array}{l}\text { A5 voltage is } 0.96 \text { p.u., } \mathrm{A} 8 \\
\text { voltage is } 1.065 \text { p.u. }\end{array}$ & $\begin{array}{l}\text { B3 voltage is } 0.95 \\
\text { p.u., B7 voltage } \\
\text { is } 1.06 \text { p.u. }\end{array}$ \\
\hline
\end{tabular}

sequences are shown in Table 1, which demonstrates how the undesired operation status is relieved step by step.

In the beginning, we evaluate the two systems $\mathrm{A}$ and $\mathrm{B}$, respectively. While simulating the manipulation control process of the system $\mathrm{A}$, there is no curtailment of $\mathrm{EV}$ station because the injection of DC interlink is not limited. While simulating the manipulation control process of the system B, the injection of DC interlink has been fixed because of the manipulation on system A. Therefore, there is about $30 \%$ system-wide EV load curtailment. Then we need to balance these two systems for the unbalanced EV curtailment. We set the $\Delta \lambda^{B}$ as $5 \%$ to generate a table of manipulation combinations. Then the step minimizing the gap of EV load curtailment between these two systems is chosen. It can be seen that the EV load curtailment of system A occurs after the balance. The active power delivered by system A to system B has been increased and the EV load curtailment of system B gets relieved.

Repeating the sequences for all time snapshots, we will be able to obtain an estimation on the operation performance of the system. The profiles of all the controllable equipments in system A are shown in Figs. 5, 6, 7, and 8, and the final statuses of both systems are given in Fig. 9.

The results are reasonable because of two reasons.

1) Various operational constraints are covered. In Fig. 5, we divided the time into 5 segments by using optimal partitioning so that the mechanical limits of LTC transformer and shunt capacitors are considered. In Fig. 6, the capacity of inverters and power factor

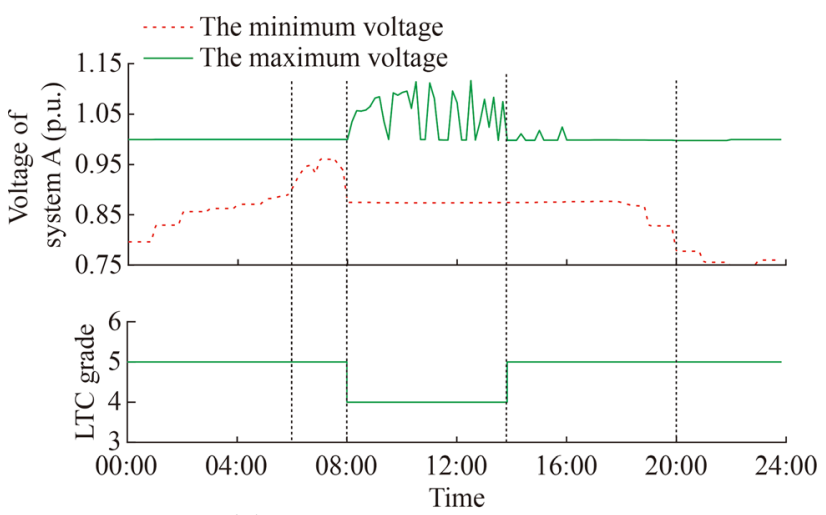

(a) Manipulation control process of LTC

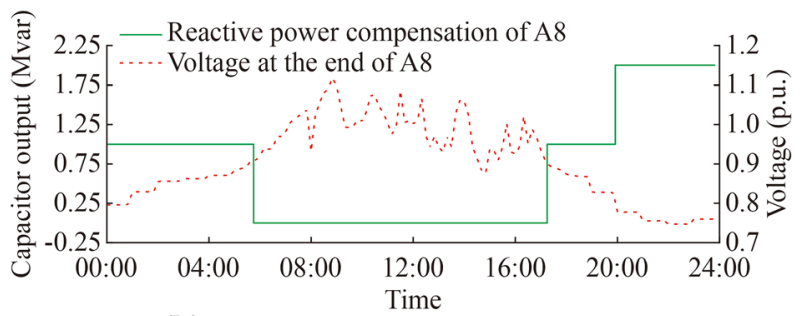

(b) Manipulation control process of capcitor

Fig. 5 Outputs of LTC transformer and capacitor in system A 

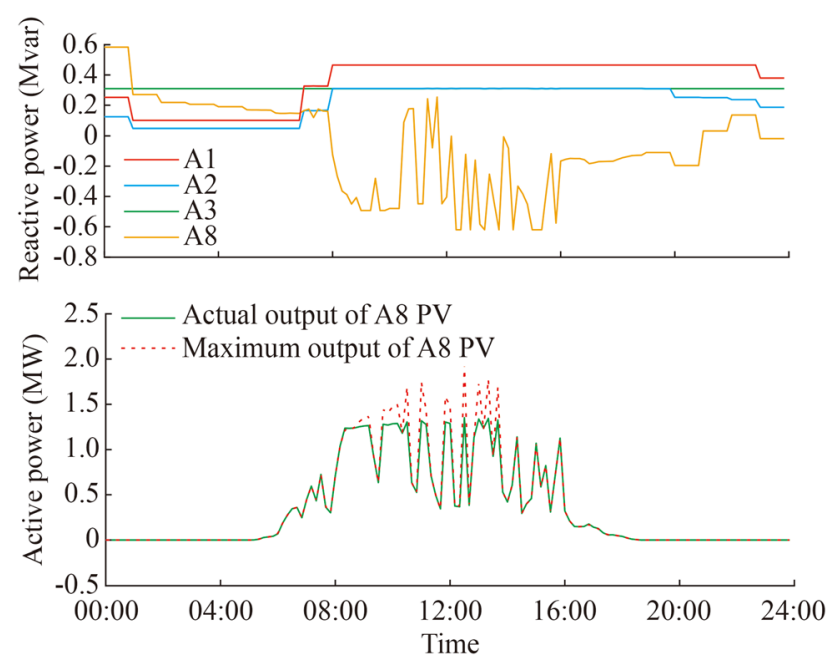

Fig. 6 Outputs of PV inverters
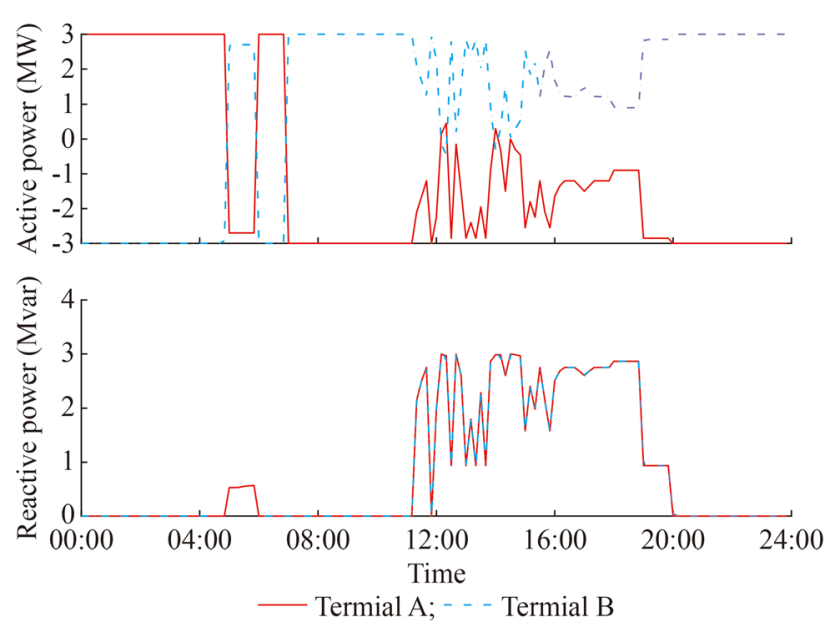

Fig. 7 Outputs of DC terminal

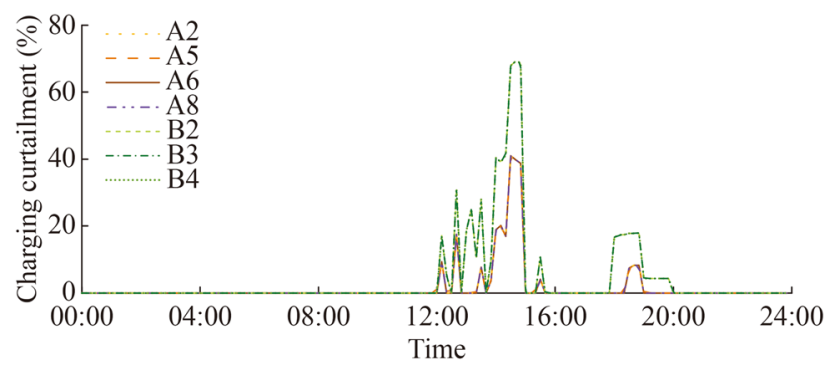

Fig. 8 Charging curtailments of EVs

constraints are satisfied. The charging load curtailment is approximately evenly distributed, which can be obtained from Fig. 8 as the curves mostly overlap each other. The charging site on A5 is curtailed more because of the voltage problem.

2) The simulation results would be one of the best possible outcomes of the system. Assuming that there

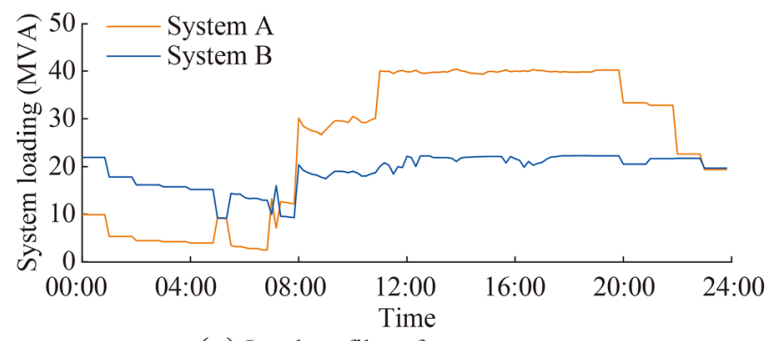

(a) Load profiles of case systems
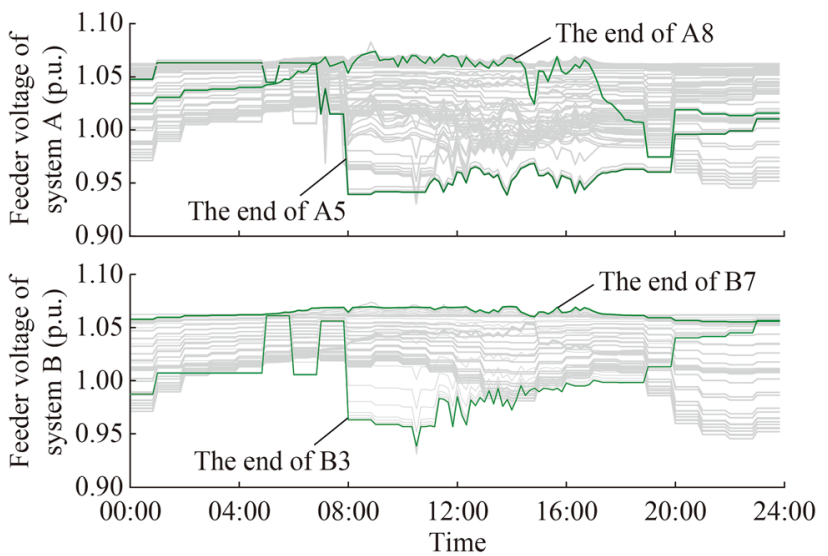

(b) Voltage profiles of case systems

Fig. 9 Simulated final status of case systems

is a better outcome that charging curtailment can be avoided on feeder A8, then we will need to tune down the voltage by reducing the secondary voltage of LTC transformer and the reactive power injection of capacitor, and letting PV inverters consume more reactive power. Adjusting the secondary voltage of LTC transformer means the overall voltage will be reduced so that feeder A5 will require more active and reactive power injections from DC interlink (which imposes more burden on system $\mathrm{B}$ ) or $\mathrm{EV}$ (which reduces successive charging percentage). Reducing the reactive power injection of capacitor means that feeder A8 will have under-voltage problems later in the same time segment. Consuming more reactive power is impossible because of the power factor and inverter capacity constraints. Therefore, the simulation is near optimal and there is no significantly better solution to the situation.

Finally, according to Fig. 9, this planning solution is sufficient considering the controllability and flexibility of distributed resources. It is not necessary to upgrade the system further.

\subsection{Comparison of computational efficiency}

Our fast algorithm can carry out the manipulation control process of a distribution system planning solution to 
Table 2 Algorithm comparison

\begin{tabular}{llr}
\hline Algorithm & Planning decision & Computation time (s) \\
\hline Proposed & 3 MVA-3 MVA & 40 \\
Bulid-in MINLP & Infeasibility declared & $>7200$ \\
Bulid-in GA & No meaningful result & $>7200$ \\
MISOCP & 3 MVA-3 MVA & 3360 \\
\hline
\end{tabular}

quantify steady-state voltages and load profiles. In this part, the significant computation efficiency advantage of the proposed algorithm is demonstrated in Table 2.

The proposed algorithm is tested against some wellknown optimal power flow optimization algorithms, including MINLP, genetic algorithm (GA) with a 10000 population size, and a mixed integer second-order cone programming (MISOCP) model. The former two are solved by Build-in MATLAB tools and the last by MOSEK and CVX toolboxes.

The problem is scanning the least capacity of the DC interlink terminals (a integer from 1 to 5 MVA) satisfying the same voltage, capacity and EV charging completion constraints, which are evaluated using either optimization or the proposed algorithm. Due to the page limitation, only the results are presented in Table 1. The MINLP and GA do not yield useful results after 2 hours of computation. The MISOCP presents a good result after 3360 s of compution. On the other hand, the proposed algorithm presents the same planning decision as the MISOCP in only $40 \mathrm{~s}$, which demonstrates the significant advantage in efficiency.

\section{Conclusion}

Future distribution systems would have complicated characteristics. The planning work of distribution systems is becoming more difficult. This paper proposes a fast algorithm to simulate the manipulation control process of controllable resources, and achieve the feasibility evaluation of a distribution system planning solution. This algorithm effectively handles five types of controllable equipment. The detailed evaluation results such as voltage, load, operations of equipment and charging completion profiles can be applied to evaluate the performance of a planning decision. Meanwhile, the algorithm breaks a large problem into a series of small ones according to the ports of the DC interlink. Each part can be solved in a carefully designed procedure. The impact of each device manipulation on the system is obtained through the flow sensitivity and the second-order power loss sensitivity instead of optimal power flow calculation. As a consequence, this algorithm has a significant efficiency advantage over some existing optimization approaches.

Acknowledgements This work was supported by National Key Research and Development Program (No. 2017YFB0903300) and National Natural Science Foundation of China (No. 51777105).

Open Access This article is distributed under the terms of the Creative Commons Attribution 4.0 International License (http:// creativecommons.org/licenses/by/4.0/), which permits unrestricted use, distribution, and reproduction in any medium, provided you give appropriate credit to the original author(s) and the source, provide a link to the Creative Commons license, and indicate if changes were made.

\section{References}

[1] Ma Z, Zhou X, Shang Y et al (2015) Form and development trend of future distribution system. Proc of CSEE 35(6):1289-1298

[2] Zhu L, Zhou X, Zhang X et al (2018) Integrated resources planning in microgrids considering interruptible loads and shiftable loads. J Mod Power Syst Clean Energy 6(4):802-815

[3] Chaudhary SK, Guerrero JM, Teodorescu R (2015) Enhancing the capacity of the ac distribution system using DC interlinks: a step toward future DC grid. IEEE Trans Smart Grid 6(4): 1722-1729

[4] Rodriguez P, Rouzbehi K (2017) Multi-terminal DC grids: challenges and prospects. J Mod Power Syst Clean Energy 5(4):515-523

[5] Liu J, Gao H, Ma Z et al (2015) Review and prospect of active distribution system planning. J Mod Power Syst Clean Energy 3(4):257-267

[6] Sheng W, Liu KY, Liu Y et al (2015) Optimal placement and sizing of distributed generation via an improved nondominated sorting genetic algorithm II. IEEE Trans Power Deliv 30(2):569-578

[7] Munoz-Delgado G, Contreras J, Arroyo JM (2015) Joint expansion planning of distributed generation and distribution networks. IEEE Trans Power Syst 30(5):2579-2590

[8] Ye X, Le J, Liu Y et al (2018) A coordinated consistency voltage stability control method of active distribution grid. J Mod Power Syst Clean Energy 6(1):85-94

[9] Pereira BR, Geraldo RMC, Javier C et al (2016) Optimal distributed generation and reactive power allocation in electrical distribution systems. IEEE Trans Sustain Energy 7(3):975-984

[10] Wu H, Huang C, Ding M et al (2017) Distributed cooperative voltage control based on curve-fitting in active distribution networks. J Mod Power Syst Clean Energy 5(5):777-786

[11] Kraiczy M, Stetz T, Braun M (2018) Parallel operation of transformers with on load tap changer and photovoltaic systems with reactive power control. IEEE Trans Smart Grid 9(6):6419-6428

[12] Hakimi SM, Moghaddas-Tafreshi SM (2014) Optimal planning of a smart microgrid including demand response and intermittent renewable energy resources. IEEE Trans Smart Grid 5(6):2889-2900

[13] Hattam L, Greetham VD (2017) Green neighbourhoods in low voltage networks: measuring impact of electric vehicles and photovoltaics on load profiles. J Mod Power Syst Clean Energy 5(1):105-116 
[14] Erdinç O, Taşcıkaraoğlu A, Paterakis GN (2018) Comprehensive optimization model for sizing and siting of DG units, EV charging stations, and energy storage systems. IEEE Trans Smart Grid 9(4):3871-3882

[15] Marano-Marcolini A, Villarejo BM, Fragkioudaki A et al (2016) DC link operation in smart distribution systems with communication interruptions. IEEE Trans Smart Grid 7(6):2962-2970

[16] Ameli A, Bahrami S, Khazeli F et al (2014) A multi-objective particle swarm optimization for sizing and placement of DGs from DG owner's and distribution company's viewpoints. IEEE Trans Power Del 29(4):1831-1840

[17] Foster DJ, Berry MA, Boland N et al (2014) Comparison of mixed-integer programming and genetic algorithm methods for distributed generation planning. IEEE Trans Power Syst 29(2):833-843

[18] Sun D, Xie X, Wang J et al (2014) Integrated generationtransmission expansion planning for offshore oilfield power systems based on genetic Tabu hybrid algorithm. J Mod Power Syst Clean Energy 5(1):117-125

[19] Ravadanegh SN, Roshanagh RG (2014) On optimal multistage electric power distribution networks expansion planning. Int $\mathbf{J}$ Electr Power and Energy Syst 54(1):487-497

[20] Sedghi M, Ahmadian A, Aliakbar-Golkar M (2016) Optimal storage planning in active distribution network considering uncertainty of wind power distributed generation. IEEE Trans Power Syst 31(1):304-316

[21] Zhao B, Hu Z, Zhou Q et al (2019) Optimal transmission switching to eliminate voltage violations during light-load periods using decomposition approach. J Mod Power Syst Clean Energy 7(2):297-308

[22] Zhao Y, Hesamzadeh RM (2019) Second-order cone AC optimal power flow: convex relaxations and feasible solutions. J Mod Power Syst Clean Energy 7(2):268-280

[23] Cui B, Sun AX (2018) A new voltage stability-constrained optimal power-flow model: sufficient condition, SOCP representation, and relaxation. IEEE Trans Power Syst 33(5):5092-5102

[24] Kocuk B, Dey SS, Sun X (2016) Inexactness of SDP relaxation and valid inequalities for optimal power flow. IEEE Trans Power Syst 31(1):642-651

[25] Bose S, Low HS, Teeraratkul T et al (2015) Equivalent relaxations of optimal power flow. IEEE Trans Autom Control 60(3):729-742
[26] Liu Y, Li J, Wu L (2019) Coordinated optimal network reconfiguration and voltage regulator/DER control for unbalanced distribution systems. IEEE Trans Smart Grid 10(3):2912-2922

[27] Willis HL (2004) Power distribution planning reference book, 2nd edn. CRC Press, Boca Raton

[28] Ayres HM, Salles D, Freitas W (2014) A practical second-order based method for power losses estimation in distribution systems with distributed generation. IEEE Trans Power Syst 29(2):666-674

[29] Cheng L, Chang Y, Huang R (2015) Mitigating voltage problem in distribution system with distributed solar generation using electric vehicles. IEEE Trans Sustainable Enery 6(4):1475-1484

[30] Cheng L, Chang Y, Wu Q et al (2014) Evaluating charging service reliability for plug-in evs from the distribution network aspect. IEEE Trans Sustain Energy 5(4):1287-1296

Lin CHENG received the Ph.D. degree in electrical engineering from Tsinghua University, Beijing, China, in 2001. Currently, he is an associate professor with the Department of Electrical Engineering, Tsinghua University, Beijing, China. His research interests are reliability of power system, power system planning and operation.

Yuxiang WAN received the B.S. degree in electrical engineering from Tsinghua University, Beijing, China, in 2017. Currently, he is pursuing the Ph.D. degree with the Department of Electrical Engineering, Tsinghua University, Beijing, China. His research interests are in the areas of demand response and power system reliability.

Liting TIAN received the B.S. and M.S. degrees in electrical engineering from Tsinghua University, Beijing, China, in 2004 and 2007, respectively. Currently, She is a senior engineer with the Department of Electrical Engineering, Tsinghua University, Beijing, China. His research interests are the planning and operation of the distribution power system.

Fang ZHANG received the Ph.D. degree in electrical engineering from Tsinghua University, Beijing, China, in 2015. Currently, he is an associate professor with School of Electrical Engineering, Beijing Jiaotong University, Beijing, China. His research interests are in the areas of power system dynamic control. 